Test Method

\title{
An adaptable flexural test fixture for miniaturised polymer specimens
}

\author{
Gabriel Y.H. Choong ", Alessia Canciani, Davide S.A. De Focatiis \\ Faculty of Engineering, University of Nottingham, Nottingham, NG7 2RD, UK
}

\section{A R T I C L E I N F O}

\section{Keywords:}

Flexural testing

Three-point bending

Miniature specimens

Mechanical properties

Hydrolytic degradation

\begin{abstract}
A B S T R A C T
An adaptable flexural test fixture is proposed to characterise the mechanical properties of miniature beam specimens ( $10 \mathrm{mg}$ ) at ambient conditions or in the presence of fluids at elevated temperatures. The fixture is validated using representative amorphous and semi-crystalline polymers. The response of miniature specimens is compared against that of medium-sized specimens ( $1 \mathrm{~g}$ ) on the same fixture and on conventional test equipment. Good agreement is found between the specimen sizes for all materials, but the comparisons highlight small differences attributed to factors such as specimen dimensional accuracy, crystallinity and span-to-thickness ratios. Flexural tests in water at $37 \mathrm{C}$ using both specimen sizes were performed to investigate the evolution of mechanical behaviour of hydrolytically degraded polylactides. Here, specimen size influences the diffusion timescale of acidic by-products which can reduce or enhance autocatalysis.
\end{abstract}

\section{Introduction}

The characterisation of the bulk mechanical properties of a polymeric material is an essential step in defining material applications and establishing performance envelopes. This can often be hampered by the limited amount of material available, such as during the development of new material chemistries or when the addition of exotic fillers into polymers is being investigated. For example, a typical laboratory benchtop compounder can process only a few grams of material. Such quantities are not sufficient for conventional protocols and standards to determine bulk properties. This means that the typical standard sample size as recommended by British Standard (EN ISO 178:2019) with dimensions of $80 \quad 10 \quad 4 \mathrm{~mm}^{3}$ [1] for conventional mechanical testing cannot be produced, and non-conventional test equipment is required to perform miniaturised mechanical tests.

Mechanical testing of miniature specimens to determine bulk properties has been pursued over the last 40 years to support material development in industries such as nuclear, power generation and aerospace, with an emphasis on metallic materials. Test methods typically used to evaluate metals, for example small punch tests, have been applied to polymeric materials but the discs specimens lead to complex stress states that are challenging to relate to conventional tensile tests. The development of miniature polymeric test specimens is commonly associated to the characterisation of microinjection mouldings and of biological samples. Constraints of tensile and flexural microtesters and of mechanical analysers (e.g. dynamic and thermomechanical) include the requirements for specific specimen shapes, significant specimen gripping challenges, limited force and displacement range, and the need for specialised equipment accessories to test in different environmental conditions $[2,3]$. The literature summarised in Table 1 reports examples of different approaches to develop test fixtures and methods for simple geometries of miniature specimens of polymeric materials to investigate mechanical behaviour, using specimens with characteristic dimensions of $15 \mathrm{~mm}$.

A comprehensive review of techniques by Lewis et al. [4] focused on the punch method to characterise miniature specimens of polymeric materials, primarily ultra-high-molecular-weight polyethylene (UHMWPE) and acrylic bone cement. The punch-type approaches can be divided into equibiaxial tensile and equibiaxial shear deformation tests, where the tests differ in the shape of the punch end, either hemispherical or flat-ended. The main disadvantages of the punch approach come from the complex contact mechanics involving compression, tension and shear, and the complexity in transforming load-displacement data into stress-strain data to yield the bulk mechanical properties. Although the transformation can be carried out by an empirical approach, an analytical solution, or via an assumed constitutive model, there is limited applicability of the approach to all experimental datasets, and limited availability of corroborating data from standard tests for bulk properties to compare against.

In 1989, Kuhn et al. adapted a Leitz microscope to house a 3-point bending fixture to measure the elastic moduli of the trabecular and cortical tissue from human iliac crests [5]. Tissues were cut into beam

\footnotetext{
* Corresponding author.

E-mail address: gabriel.choong@nottingham.ac.uk (G.Y.H. Choong).
} 
specimens with a span length-to-thickness $(L / d)$ ratio of $\sim 12: 1$ and supported across a span of $1.04 \mathrm{~mm}$, on the microscope stage. The selected ratio is near to the British Standard recommended $L / d$ ratio of 16, decreasing to 8 for thin specimens [1]. A correction factor for shear effects due to local stress perturbations was considered in the calculation of the modulus. Control flexural tests performed with silicon carbide fibres were found to be within $10 \%$ of the manufacturer's reported moduli.

Chaplin et al. employed a commercial micro-tester to perform 3point bending tests horizontally in an effort to study the progression of mechanical properties of recovered polymethylmethacrylate (PMMA) bone cement from hip prostheses taken during patients' revision surgeries, to shed insights about the cement's service life [6]. The elastic and stress relaxation moduli of PMMA cement were determined in both wet and dry conditions. Specimens were cut with a span $L / d$ ratio ranging from $\sim 7$ up to $\sim 10$. Studies compared the effect of the shape of the specimen supports, and both flat $1 \mathrm{~mm}$ wide supports with rounded edges and rounded supports with a radius of $0.95 \mathrm{~mm}$ showed similar results. The authors selected the flat supports for their work, with a typical span between supports of $10.3 \mathrm{~mm}$, and a loading nose tip width of $0.18 \mathrm{~mm}$. The load cell had a capacity of up to $4 \mathrm{~N}$ and a pre-load of $0.4 \mathrm{~N}$ was applied to all specimens to hold it in position prior to testing. The relatively low load capacity limits the type of materials that the equipment can investigate, and positioning a specimen for horizontal loading can be challenging. Their work used linear elastic beam theory to calculate the elastic modulus but neglected the effects of shear and local deformations, as well as of bending rotations.

In order to evaluate the mechanical properties of cortical osteotomy specimens to understand genetic disorder of brittle bones, Albert et al. developed a 3-point bending fixture that couples with a universal testing machine [7]. The challenge faced was that surgical paediatric bone specimens typically collected are limited in length $5 \mathrm{~mm}$ and hence a span length $L \quad \sim 4 \mathrm{~mm}$ was used. Due to the microstructural features of the material, Albert et al. employed an $L / d$ of $\sim 5$ to 6 , that is, in close proximity to the British Standards suggestion for thin specimens [1], and specimens thicknesses ranged from $\sim 0.65$ to $0.80 \mathrm{~mm}$. The device was validated on acrylic and bovine bones prior to testing osteotomy samples. The authors acknowledged that local deformations were not considered, and linear elastic behaviour was assumed throughout the test, likely leading to an underestimation of the elastic modulus and an overestimation of the material strength.

In this study we propose a test fixture to determine mechanical properties that provides (1) a simple path to the constitutive response (i. e. stress-strain data) without the need for complex transformations or model fitting, and (2) the versatility of testing in terms of specimen dimensions and environmental conditions, for example, the submerging the specimen in fluids across a range of temperatures. Firstly, we describe the development of an adaptable flexural test fixture (AFTF) to perform 3-point bending tests on miniaturised polymeric samples in dry and wet environments, and the determination of the elastic modulus with corrections related to shear deformations and bending rotations. Validation of the AFTF is carried out using measurements on both amorphous and semi-crystalline polymers using medium-sized specimens measured on the AFTF and on conventional equipment, in both ambient and wet environments. Finally, the mechanical response of accelerated hydrolytically degraded polylactides is compared between measurements performed on miniature and medium-sized specimens in water at $37 \mathrm{C}$, using the AFTF and conventional equipment correspondingly. This work is part of a wider effort to develop bioresorbable polylactides filled with hydroxyapatite nanoparticles for healthcare applications, particularly orthopaedic devices such as bone fixation screws.

\section{Experimental}

\subsection{Materials}

The materials used in this study are NatureWorks 2003D semicrystalline poly-L-lactic acid (PLLA), Evonik LR 706S amorphous poly (L-lactide-co-D, L-lactide) (PDLLA) and Dow Chemical GP-PS680E

Table 1

Examples of test equipment for miniature specimens employed to measure mechanical properties of polymeric materials with simple geometries.

\begin{tabular}{|c|c|c|c|c|}
\hline Test equipment & Test description & Properties investigated & Specimen dimensions & Reference \\
\hline $\begin{array}{l}\text { Small punch; punch head configuration } \\
\text { or shape varies between } \\
\text { hemispherical, flat-ended, concave } \\
\text { tip and metal ball. }\end{array}$ & $\begin{array}{l}\text { The punch pushes through a disc-shaped or } \\
\text { rectangular-shaped specimen along its axis of } \\
\text { symmetry, at a constant displacement rate. The punch } \\
\text { load and its displacement are recorded. }\end{array}$ & $\begin{array}{l}\text { Modulus, Work to failure (i.e. } \\
\text { Energy), Ultimate tensile } \\
\text { strength (UTS), Fracture } \\
\text { toughness }\end{array}$ & $\begin{array}{l}\text { Typical disc dimensions: } \varnothing 3 \\
\text { to } 20 \mathrm{~mm} \text {; thickness } 0.1 \text { to } \\
1.0 \mathrm{~mm} \text {. } \\
\text { Typical square dimensions: } \\
\text { width } 4 \text { to } 10 \mathrm{~mm} \text {; thickness } \\
\quad 0.25 \text { to } 0.5 \mathrm{~mm}\end{array}$ & [4] \\
\hline $\begin{array}{l}\text { 3-point bending fixture constructed } \\
\text { from a microscope body without } \\
\text { optics }\end{array}$ & $\begin{array}{l}\text { The loading nose was attached to the microscope body } \\
\text { tube and a stepper motor, connected to the fine focus, } \\
\text { is used to control the vertical motion of the stage. A } \\
\text { load cell and specimen supports are fixed on the stage } \\
\text { in series. Force and displacement are recorded using a } \\
\text { load cell and a linear variable differential transformer } \\
\text { (LVDT) respectively. }\end{array}$ & Modulus & $\begin{array}{l}\text { Typical beam dimensions: } \\
\text { width and thickness } 0.05 \text { to } \\
0.2 \mathrm{~mm} \text {; length } 1.5 \text { to } 2.0 \\
\mathrm{~mm}\end{array}$ & [5] \\
\hline Horizontal 3-point bending & $\begin{array}{l}\text { A commercial microtester (manufacturer not specified) } \\
\text { consists of specimen supports that moves horizontally } \\
\text { on a base unit towards or away from a fixed positioned } \\
\text { loading nose that is attached in series to a load cell. } \\
\text { Wet tests can be performed by positioning a water } \\
\text { dripper over the specimen to keep it moist throughout } \\
\text { a test. }\end{array}$ & Modulus, Stress relaxation & $15 \quad 5.0 \quad 1.0 \mathrm{~mm}^{3}$ & {$[6]$} \\
\hline $\begin{array}{l}\text { Custom fixture mounted onto a } \\
\text { universal tensile testing machine in } \\
\text { compression mode }\end{array}$ & $\begin{array}{l}\text { The custom fixture consisted of top and bottom platen } \\
\text { assemblies. The top assembly is attached to the } \\
\text { machine crosshead and holds the load cell, loading } \\
\text { nose, a LVDT and four alignment pins. These pins help } \\
\text { to ensure that the loading nose is centred and parallel } \\
\text { to the specimen supports. The bottom platen contains } \\
\text { the specimen supports, holes for the pins and a } \\
\text { protruding steel plate to set the LVDT's initial position. } \\
\text { The LVDT was used to reduce displacement errors } \\
\text { attributed to the compliance of the load frame, load } \\
\text { cell and fixture. }\end{array}$ & Modulus, UTS & $\sim 4.0 \quad 1.0 \quad(0.6$ to 0.8$) \mathrm{mm}^{3}$ & [7] \\
\hline
\end{tabular}


polystyrene (PS).

Specimens were directly moulded into either flat sheets with $0.5 \mathrm{~mm}$ thickness for cutting into miniature specimens, or medium-sized beams with dimensions of $6052 \mathrm{~mm}^{3}$, by compression moulding with custom-made flash moulds [8] using a Daniels hydraulic heated press. The top and bottom surfaces of the mould were lined each time with a $0.15 \mathrm{~mm}$ thick aluminium foil to provide a repeatable surface roughness. The mould and aluminium foil were cleaned with acetone prior to the application of a light coating using PTFE spray to assist the release of the specimens from the mould. For hygroscopic polylactides, pellets were dried in a vacuum oven at $50 \mathrm{C}$ for a minimum of 12 hours prior to compression moulding. The moulding procedure consisted of a $\sim 10$ minute warm up period to reach the moulding temperature $T(170 \mathrm{C}$ for PDLLA and PS, 210 C for PLLA), a 3 minute stage where pressure was applied and released repeatedly to dislodge any trapped air, holding for a further 3 minute at $T$ to allow for polymer relaxation, and a cooling stage where cold water is flushed through channels in the heated platens, producing a repeatable cooling rate of $\sim 20 \mathrm{C} \mathrm{min}{ }^{1}$ through to a temperature sufficiently below $T_{\mathrm{g}}$.

\subsection{Miniature specimen preparation}

Each polymeric sheet of $0.5 \mathrm{~mm}$ thickness was produced with less than $1 \mathrm{~g}$ of polymer. Miniature beam specimens with parallel sides were cut from $0.5 \mathrm{~mm}$ sheets using a custom jig with parallel single-bevelled blades [9]. The strips were then trimmed to length, producing rectangular prismatic beams typically with dimensions of $7 \quad 2 \quad 0.5 \mathrm{~mm}^{3}$. The long axis of the beams was aligned with the rolling direction of the aluminium foil mould liner to give the specimens a reproducible surface roughness. A batch of miniature beam specimens were immersed in phosphate buffer solution (PBS) and stored at $50 \mathrm{C}$ for a period ranging from 4 hours up to 21 days.

\subsection{Adaptable flexural test fixture}

The AFTF assembly, displayed in Fig. 1, is a modification of a miniature creep fixture developed by De Focatiis and Buckley for the determination of craze initiation stress in miniature polymer specimens [9]. A linear motion actuator with an integrated controller (CONEX-LTA-HS, Newport Corp.) was mounted on a large micrometer lead screw for calibration and general positioning; and replaced the original
LVDT in the creep rig. The actuator is connected co-axially with a miniature tension/compression load cell (Omega Engineering Ltd.). Two separate load cells were used depending on the specimen size: a load cell with a force range of up to $10 \mathrm{~N}$ was employed for miniature beams, whereas a $100 \mathrm{~N}$ capacity load cell was used with medium-sized beams. The loading noses for the miniature and medium-sized specimens have an edge radius of approximately $0.6 \mathrm{~mm}$ and $2.4 \mathrm{~mm}$ respectively. When the actuator is directed towards the specimen, it applies a load through the loading nose that is recorded via the load cell. LabVIEW (National Instruments) software was employed to control the actuator to move at a prescribed speed and to acquire both load and displacement data.

For miniature beams, adjustable supports are mounted on small micrometer lead screws. These supports have a step with a curved edge with a radius of $\sim 0.25 \mathrm{~mm}$. The beam specimen is located on the supports by placing the end of the beam against the flat face of the support step. The distance between the supports is typically set at $5 \mathrm{~mm}$ and can be extended up to $10 \mathrm{~mm}$. For medium-sized specimens, a sub-stage with roller supports of radius of $2.5 \mathrm{~mm}$ is fixed into position. The support positions are adjustable to provide three distances between the rollers of $25 \mathrm{~mm}, 50 \mathrm{~mm}$ and $80 \mathrm{~mm}$. A distance of $50 \mathrm{~mm}$ between the rollers was used in this work. In order to ensure that the loading nose is located at the centre of the specimen, a locating tool with notches at predetermined positions is used to assist the location of the sub-stage and the rotation angle of the loading nose.

Sources of experimental error can arise from both measurement of load and of displacement. The load cell is calibrated independently using known weights. The load calibration was performed with the load cell rotated through 180 and a custom load tray to support the weights; the relative weights of the loading noses and tray were accounted for during the calibration. The linearity of voltage measurement from the load cell with respect to applied force was measured, and a coefficient of determination $R^{2} \quad 0.99$ was obtained. The displacement of the actuator rod was compared with the displacement of the independent large micrometer lead-screw, and actuator position was within $0.02 \%$ of micrometer displacement over the measurement range.

Compliance of the fixture for the set-ups to perform flexural testing on two specimen geometries was determined using a stiff metal block, and the fixture compliance is shown in Fig. 2. The maximum axial load capacity for the actuator is $40 \mathrm{~N}$ according to the manufacturer, and hence compliance calibration was carried out below the upper load limit of the $100 \mathrm{~N}$ capacity load cell. Compliance calibration for the miniature
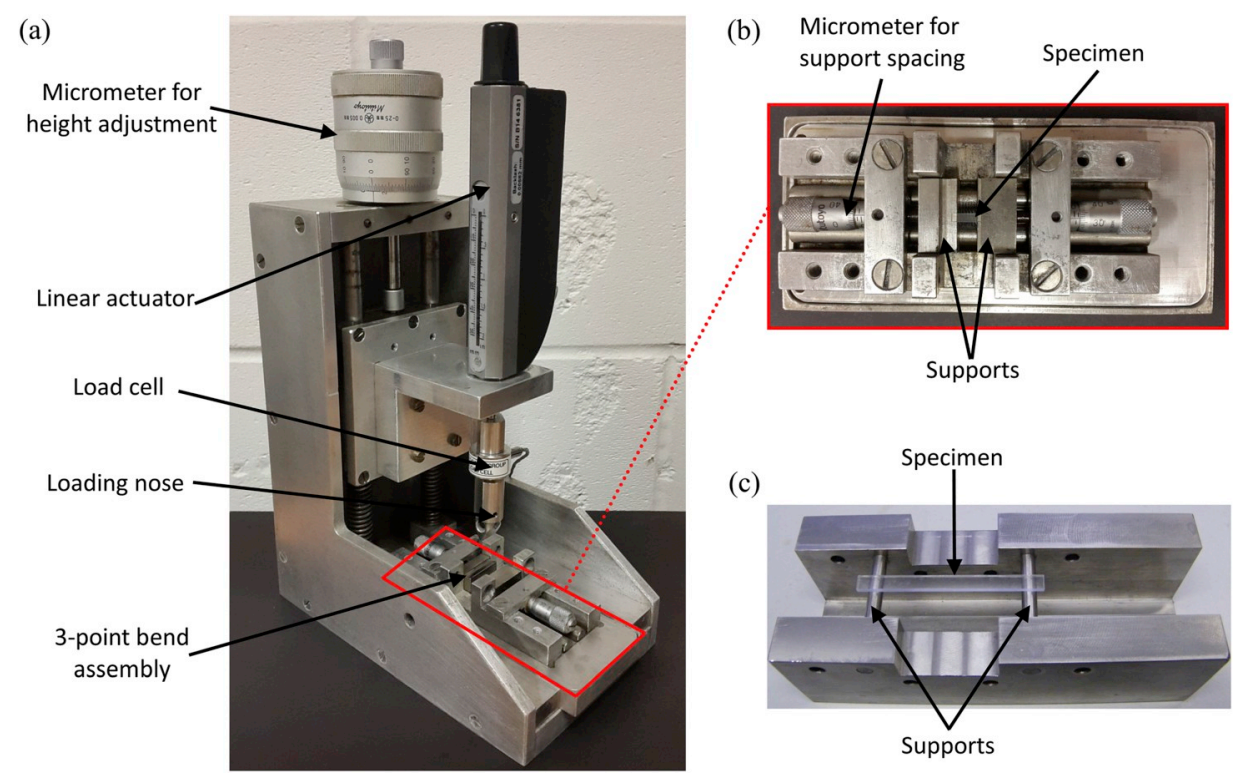

Fig. 1. (a) The adaptable flexural test fixture (AFTF) with the miniature specimen supports. Top view of (b) the supports for a miniature beam specimen (7 $2 \quad 0.5$ $\mathrm{mm}^{3}$ ) and (c) of the supports for medium-sized beam specimens $\left(\begin{array}{lll}6 & 5 & 2\end{array} \mathrm{~mm}^{3}\right.$ ). 


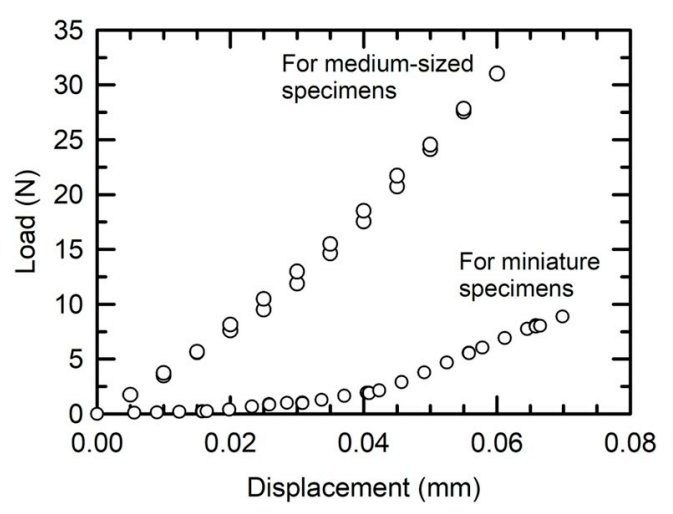

Fig. 2. AFTF compliance for the miniature specimen and the medium-sized beam set-up with the $10 \mathrm{~N}$ and $100 \mathrm{~N}$ capacity load cells respectively.

specimen was conducted over the full range of the $10 \mathrm{~N}$ capacity load cell.

For the medium-sized specimen associated with the $100 \mathrm{~N}$ capacity load cell, a second-order polynomial was sufficient to capture the compliance data. In the case of the miniature specimen, the correction to the recorded displacement was carried out by linear interpolation using the compliance data. For both specimen sizes, the true displacement for each flexural measurement was determined by subtracting the fixture's compliance, based on the recorded load of a specific test, from the displacement of the actuator.

Owing to the size of the miniature specimens, slight specimen geometry surface defects can lead to partial contact between the loading nose and the specimen, thus affecting the load measurements at very low strains. Representative raw load-displacement data is shown in Fig. 3(a) for a PLLA miniature specimen at ambient conditions both before and after the compliance correction. Even after the compliance correction, at small displacements the displacement does not always increase monotonically with load. In order to correct the former, linear regression was carried out on load-displacement data for loads $0.8 \mathrm{~N}$, and extrapolated to identify the displacement at zero force. Starting with the smallest force, data points were iteratively discarded until the $R^{2}$ of the linear regression reached 0.99 . At this point, the correct displacement at zero force could be determined and the data shifted along the displacement axis to produce a curve passing through the origin. This process is illustrated in the inset of Fig. 3(a). The extrapolated values are used for display purposes only and were not considered in the subsequent determination of the elastic modulus. Finally, the entire corrected load-displacement curve is illustrated in Fig. 3(b).

The stress-strain data is directly determined from the corrected loaddisplacement data using the specimen cross-sectional area and test span length. Flexural stress $\sigma$ for a rectangular cross section beam is calculated from the measured load, given as $\sigma \quad \frac{3 P L_{0}}{2 b d^{2}}$ where $P$ is the measured force, $L_{0}$ is the initial test span length, $b$ is the width of the specimen, $d$ is thickness of the specimen. Flexural strain is determined from the controlled displacement given by $\varepsilon \quad \frac{6 \delta_{c} d}{L_{0}^{2}}$ where $\delta_{c}$ is the deflection of the centre of the beam after correction for fixture compliance. Therefore, the gradient of the stress-strain curve provides the experimentally determined modulus, $E_{\mathrm{f}}$, without additional corrections for the effects of shear, rotation at the supports, and local contact deformations.

For testing in the presence of fluids at a range of temperatures, the entire AFTF can be placed in a temperature-controlled fluid bath. Precautionary steps need to be taken to ensure that electrical connections for the actuator and load cell are not submerged in the fluid. The miniature beam supports within the 3-point bend assembly (as shown in Fig. 1(b)), are immersed in a water bath set to 37 C. A minimum acclimatisation time of 30 minutes was applied prior to the start of each test to allow the water bath and the fixture to reach a steady temperature. The temperature of the water during testing was monitored with a (a)

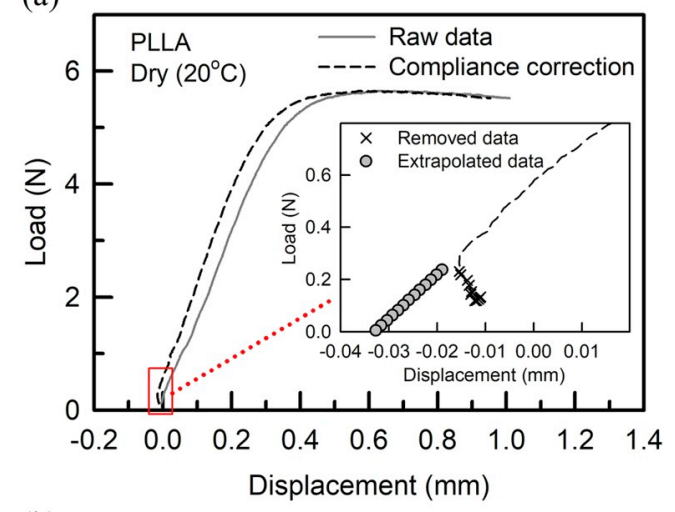

(b)

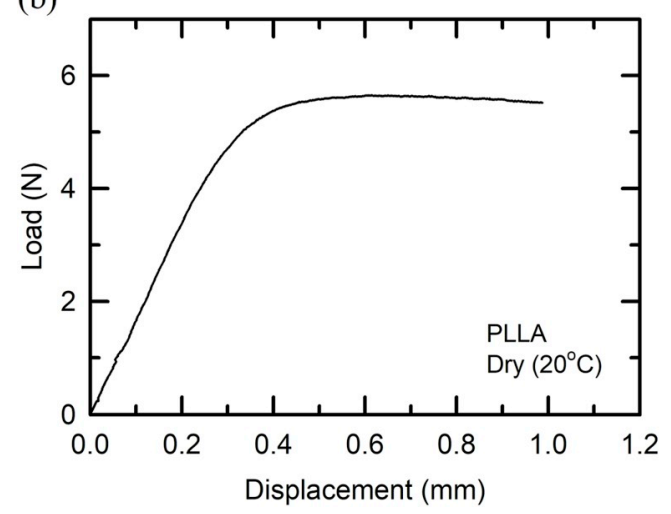

Fig. 3. (a) Representative raw load-displacement data for a PLLA miniature specimen ( $\left.\begin{array}{lll}7 & 2 & 0.5 \mathrm{~mm}^{3}\end{array}\right)$ tested with the AFTF, and the compliance corrected load displacement data. Inset shows the method of determining the true displacement at zero force. (b) Load-displacement curve corrected for both compliance and displacement offset.

K-type thermocouple positioned near the specimen.

\subsection{Determination of elastic modulus}

When performing 3-point bending tests, there is a need for consideration of (1) shear effects due to span length (arising from small $L / d$ ratios), (2) bending rotations attributed to finite support dimensions, and (3) local contact deformation occurring at the supporting rollers and loading nose. These considerations were factored into an approach proposed by Mujika to correct 3-point bending measurements for carbon fibre composites [10]. Mujika assumed that local deformations of a specimen at the supports and loading nose could be modelled as linear springs, resulting in the corrected modulus $E$ as [10].

$\begin{array}{llllllllll}E & E_{f} & 1 & \frac{3 R}{d} \bar{\varepsilon} & \frac{6}{5 G} & 1 & \frac{R}{d} \bar{\varepsilon} & {\frac{d}{L_{0}}}^{2} & \frac{4 b}{k} & {\frac{d}{L_{0}}}^{3}\end{array}$

where $E_{\mathrm{f}}$ is the experimentally determined flexural modulus, $R$ is the radius of cylindrical supports, $\bar{\varepsilon}$ is the strain range, $G$ is the shear modulus and $k$ are spring constants for the specimen supports and loading nose. The third term in Equation (1) relates to local deformation at the support rollers and loading nose, and it is suggested that the contact stiffness $k$ can be measured experimentally via indentation tests. Mujika's indentation tests of carbon fibre specimens, which is dominated by epoxy resin, recorded values of $k \simeq 10^{4} \mathrm{~N} \mathrm{~mm}{ }^{1}$. Assuming a similar $k$ for thermoplastics, the contribution of the third term to the modulus correction is negligible considering the specimen geometry employed in this work. 
Given the relationship between elastic constants $G \quad \frac{E_{f}}{21 v}$ where $v$ is the Poisson's ratio of the material, Equation (1) can be written as

$E \quad E_{f} \quad 1 \quad \frac{3 R}{d} \bar{\varepsilon} \quad \frac{121 \quad v}{5} \quad \frac{d}{L_{0}}{ }^{2} 1 \frac{R}{d} \bar{\varepsilon}$

In this work, values of Poisson's ratio were obtained from literature as 0.34 and 0.36 for PS and polylactides respectively [11,12].

\subsection{Mechanical testing}

The width and thickness of each specimen were measured at five approximately equidistant locations, along the centreline of the length of the specimen. Measurements were carried out using a digital micrometer in the thickness direction, and a calibrated scanner system in the width direction for the miniature beams, whereas medium-sized beams were measured in both directions using a digital micrometer.

All tests were performed at a fixed cross-head displacement rate corresponding to a maximum nominal strain rate of $0.47 \quad 10^{3} \mathrm{~s}{ }^{1}$. Dry tests were performed at laboratory conditions of $\left(\begin{array}{lll}20 & 2\end{array}\right)$ C, whereas wet tests were performed in water at a temperature of $\left(\begin{array}{lll}37 & 1\end{array}\right)$ C. A minimum of four specimens were tested for each condition.

For comparison, flexural testing was also performed with commercially available test equipment in order to validate the results obtained using the AFTF. No single test fixture met all test requirements comprehensively, and tests were limited by either specimen geometry or environmental conditions. For validation of the medium-sized beams in ambient conditions, tests were carried out on two different test machines: (1) an Instron 5569 testing machine fitted with a flexural testing fixture with load and support rollers of $2.5 \mathrm{~mm}$ radius and a $5 \mathrm{kN}$ load cell and (2) Bose ElectroForce Series II 3330 with load and support rollers of $5 \mathrm{~mm}$ radius and a $3 \mathrm{kN}$ load cell. The latter equipment was also employed to carry out flexural tests in wet conditions at $37 \mathrm{C}$. The test strain rates and spacing between the rollers were matched between all the test equipment.

Elastic modulus $E$ was determined for a strain range between 0 and $1 \%$ strain for all materials using Equation (2). The flexural yield strength, $\sigma_{\mathrm{ys}}$ was extracted as the maximum engineering stress. Error bars shown in the results represent two standard errors.

\section{Results}

\subsection{Comparisons across different test equipment with medium-sized} beams

Fig. 4 presents stress-strain curves for medium-sized beam specimens of PLLA, PDLLA and PS measured at ambient conditions using the full range of test equipment. Calculations of $E$ and $\sigma_{\mathrm{ys}}$ are reported as means two standard errors in Table 2. The flexural response of PLLA obtained from the different test fixtures exhibit overlap of data in the elastic region, although $\sigma_{\mathrm{ys}}$ varies by up to $7 \%$. In the case of PDLLA, the Instron measurements of stress were consistently lower than both the AFTF and the Bose, and this is attributed to a small ambient temperature difference on the day of testing. The amorphous PDLLA is more sensitive to temperature due to its close proximity to $T_{\mathrm{g}}$ contributing to the increase in variability of data; the properties of PS and the semi-crystalline PLLA are less temperature sensitive at the same conditions. Using the Instron equipment as the benchmark, the variation in $E$ of medium-sized beams for all materials arising from different test equipment ranged from $6 \%$ to $16 \%$, while variation in $\sigma_{\mathrm{ys}}$ ranged from $9 \%$ to $13 \%$. No measurements with the Bose equipment were available for PS. On balance and considering the size of the error bars indicating sample to sample variability, flexural properties of medium-sized beams were considered reproducible with the different test equipment.
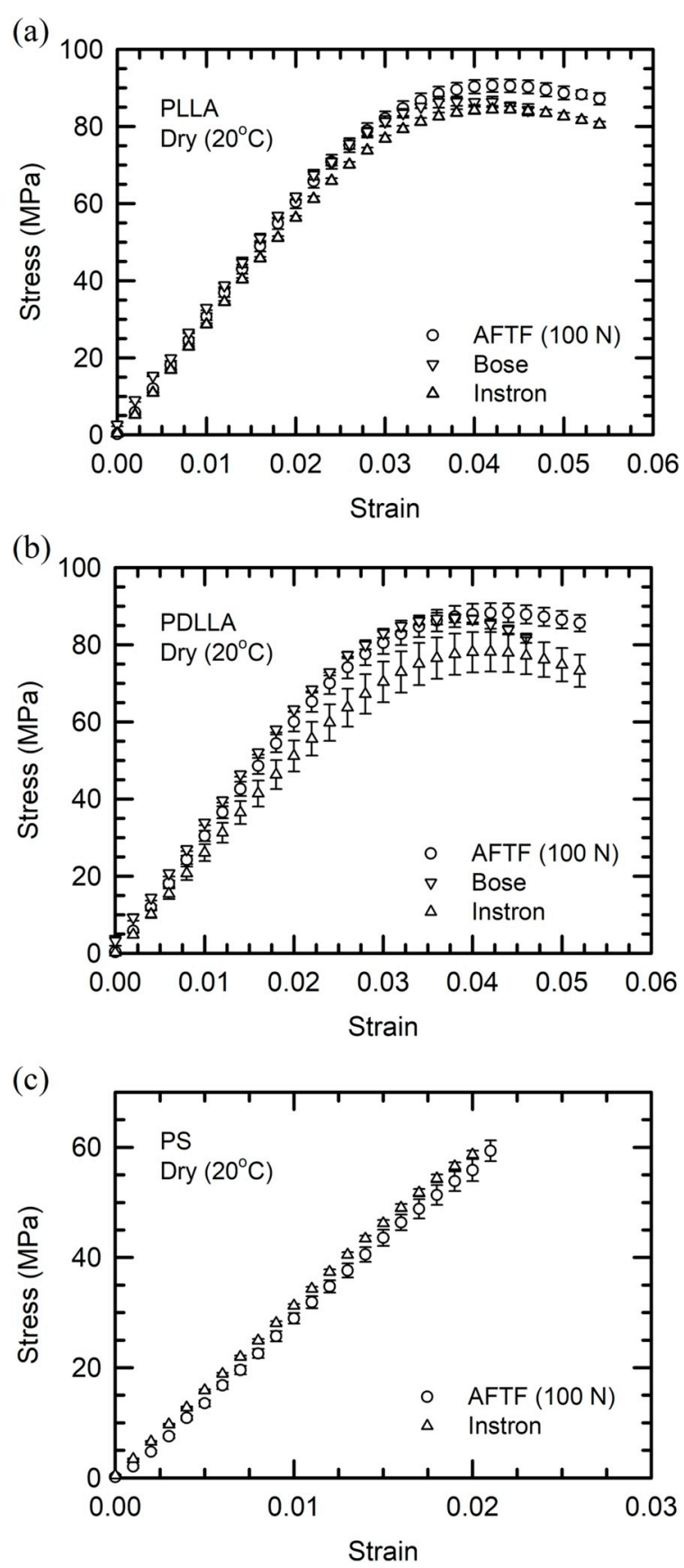

Fig. 4. Stress-strain response of medium-sized beam specimens $\left(\begin{array}{lll}60 & 5 & 2\end{array}\right.$ $\mathrm{mm}^{3}$ ) of (a) PLLA, (b) PDLLA and (c) PS determined using three different equipment (except for PS where only the AFTF and Instron were employed) at ambient conditions.

\subsection{Comparison of different specimen sizes on the AFTF}

Fig. 5 compares the stress-strain response of PLLA, PDLLA and PS determined using the AFTF on both miniature and medium-sized specimens. Table 2 reports average $E$ and $\sigma_{\mathrm{ys}}$ values, from which it can be observed that the averages obtained on the different specimen sizes are similar but not identical, with miniature specimens recording 4\%,

$14 \%$ and $2 \%$ in $E$ for PLLA, PDLLA and PS respectively. Discrepancies between the specimen sizes are observed at higher strains $(>3 \%)$, with miniature specimens consistently producing higher stress levels compared to medium-sized specimens. The reduction of specimen size resulted to an increase in $\sigma_{\mathrm{ys}}$ of $12 \%, 14 \%$ and $13 \%$ for PLLA, 
Table 2

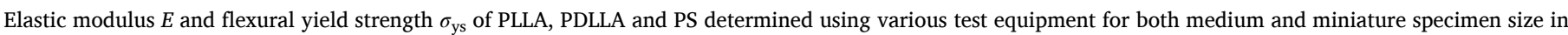
ambient conditions.

\begin{tabular}{|c|c|c|c|c|c|c|c|c|c|c|c|c|c|c|c|c|}
\hline \multirow{3}{*}{$\begin{array}{l}\text { Equipment/Material } \\
\text { PLLA }\end{array}$} & \multicolumn{8}{|c|}{$E(\mathrm{MPa})$} & \multicolumn{8}{|c|}{$\sigma_{\mathrm{ys}}(\mathrm{MPa})$} \\
\hline & \multicolumn{2}{|c|}{ Instron } & \multicolumn{2}{|l|}{ Bose } & \multicolumn{2}{|c|}{ AFTF (medium) } & \multicolumn{2}{|c|}{ AFTF (miniature) } & \multicolumn{2}{|c|}{ Instron } & \multicolumn{2}{|l|}{ Bose } & \multicolumn{2}{|c|}{ AFTF (medium) } & \multicolumn{2}{|c|}{ AFTF (miniature) } \\
\hline & 2745 & 31 & 2805 & 85 & 2951 & 82 & 3069 & 187 & 84.7 & 0.9 & 88.4 & 0.9 & 90.8 & 1.7 & 101.9 & 4.8 \\
\hline PDLLA & 2515 & 215 & 2864 & 194 & 2920 & 123 & 3336 & 466 & 78.4 & 5.1 & 88.6 & 1.2 & 88.4 & 2.5 & 100.8 & 4.1 \\
\hline PS & 2977 & 26 & $\mathrm{n} / \mathrm{a}$ & & 2795 & 97 & 2740 & 298 & 60.9 & 1.0 & $\mathrm{n} / \mathrm{a}$ & & 55.3 & 5.1 & 62.8 & 3.7 \\
\hline
\end{tabular}

(a)

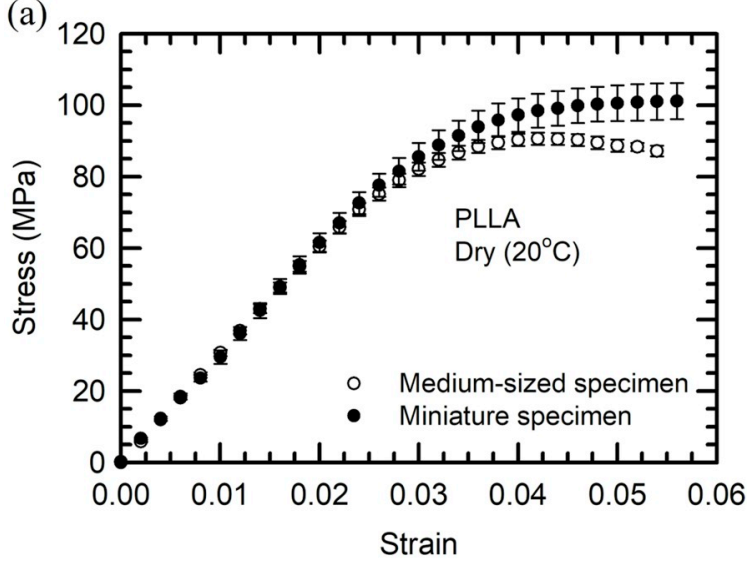

(b)

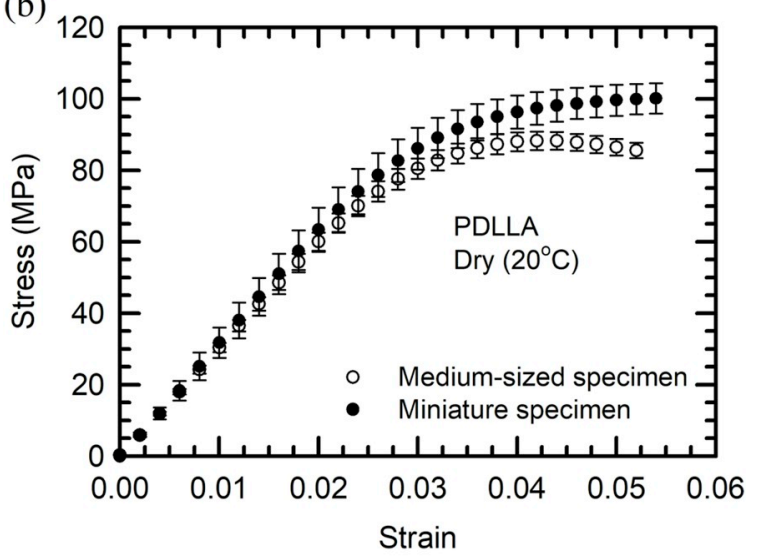

(c)

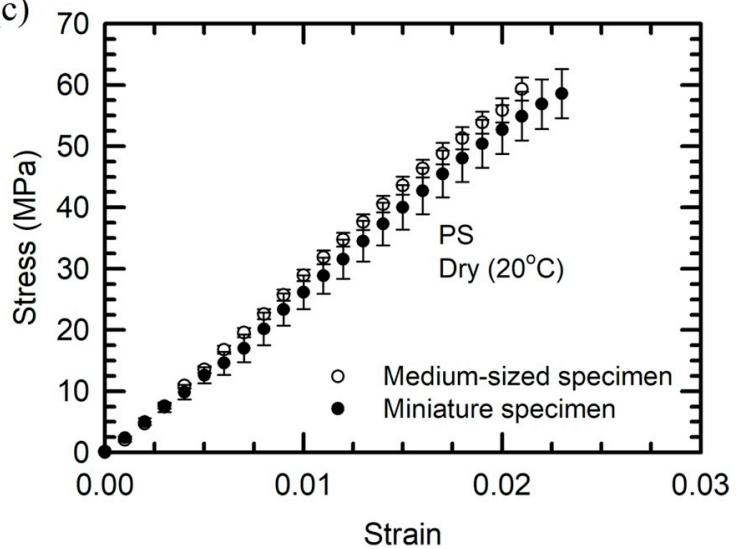

Fig. 5. Stress-strain response of medium-sized beam specimens and of miniature beam specimens of (a) PLLA, (b) PDLLA and (c) PS determined using AFTF at dry ambient conditions.
PDLLA and PS respectively.

\subsection{Comparisons of wet 37 C testing}

Fig. 6 shows a comparison of measurements of mean stress as a function of strain on polylactides in wet conditions at $37 \mathrm{C}$ between miniature specimens tested using the AFTF and medium-sized beams tested using the Bose equipment. The reduction in specimen geometry resulted in the decrease of $E$ for PLLA by $4 \%$ from $2638 \quad 84 \mathrm{MPa}$ to $2530161 \mathrm{MPa}$. However, $\sigma_{\mathrm{ys}}$ for PLLA medium-sized and miniature specimens was measured as $46.7 \quad 1.1 \mathrm{MPa}$ and $47.4 \quad 1.8 \mathrm{MPa}$ respectively, within the experimental uncertainty. For PDLLA, $E$ for medium-sized and miniature was measured as $2854 \quad 82 \mathrm{MPa}$ and 2387

$235 \mathrm{MPa}$ correspondingly, whereas $\sigma_{\mathrm{ys}}$ was determined as 55.30 .3 MPa and 44.3 1.7 MPa. Here, the change in specimen geometry led to a difference of $16 \%$ and $\quad 20 \%$ for $E$ and $\sigma_{\mathrm{ys}}$ respectively. Comparing the results for polylactides, the presence of water and the raised temperature influenced the effect of specimen dimensions on the mechanical properties of amorphous PDLLA much more so than in the semi-
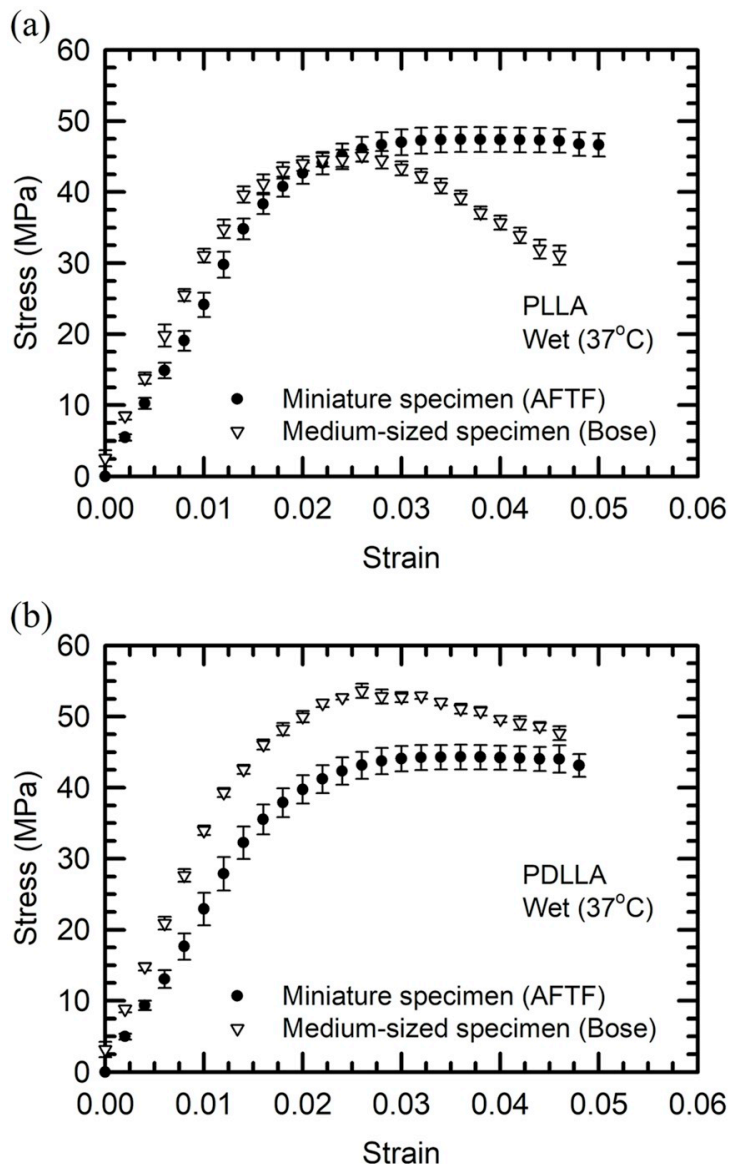

Fig. 6. Stress-strain response of miniature specimens and medium-sized specimens, determined using two different equipment in wet conditions at $37 \mathrm{C}$ for (a) PLLA and (b) PDLLA. 
crystalline PLLA.

\subsection{Comparisons of wet 37 C testing of accelerated hydrolytically degraded polylactides}

Fig. 7 shows $E$ for polylactides of different specimen dimensions that were submitted to accelerated degradation in PBS at 50 C for up to 21 days prior to flexural testing in water at $37 \mathrm{C}$. It should be noted that for the results plotted in Fig. 7, a time of $1 \mathrm{~s}$ represents as-moulded specimens with no hydrolytic degradation. Intervals for the degradation period were 4 hours, 24 hours, 5 days, 8 days, 13 days and 21 days; not all materials were characterised for the full duration of the degradation period due to specimen brittleness. Two test fixtures were employed for this study: the medium-sized beams were measured using the Bose and the miniature beams using the AFTF. Specimen beams were immersed in water and tested after a short hold time (typically a few minutes) to allow the temperature of the beam to equilibrate to the environment.

The evolution of $E$ for PLLA with degradation period presented in Fig. 7(a) revealed a trend of an initial decrease in $E$ at the start of degradation, to a plateau, and a subsequent increase after 5 days of degradation, regardless of specimen geometry and equipment. The moduli for medium-sized specimens for the degradation period ranged between 1946 and $2486 \mathrm{MPa}$, and those of miniature specimens between 2151 and $2805 \mathrm{MPa}$. The medium-sized beams recorded a noticeable increase in $E$ of $29 \%$ between day 5 and day 8 whereas miniature samples showed a similar change in $E$ of $20 \%$ between day 13 and day 21. For PDLLA, the general trend exhibited in Fig. 7(b) by the
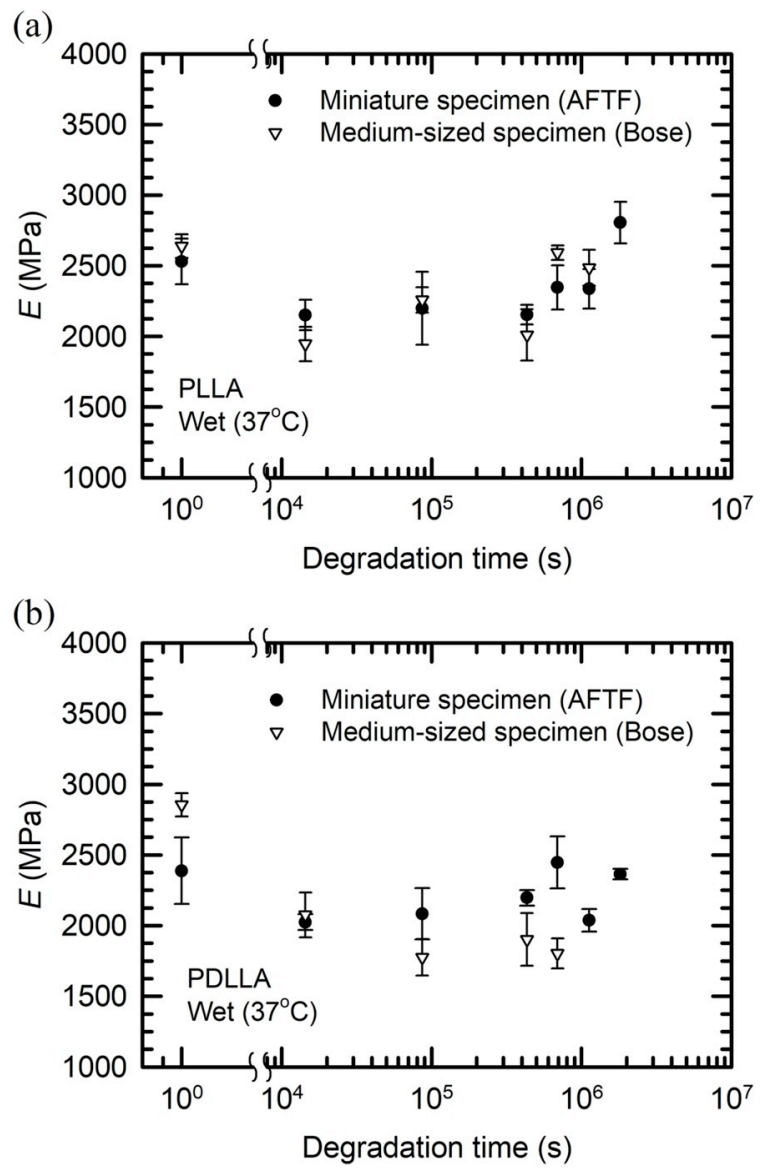

Fig. 7. Elastic modulus of medium-sized and miniature specimens for (a) PLLA and (b) PDLLA measured at $37 \mathrm{C}$ in water, determined with two different equipment, after an accelerated degradation period of up to 21 days in PBS at 50 C. Results at $1 \mathrm{~s}$ are control samples that have not experienced hydrolytic degradation. two separate measurements was similar, with an initial decrease of $E$ at the start of degradation to an approximate plateau. Moduli measurements of PDLLA medium-sized specimens are limited to 8 days of degradation. The moduli for medium-sized specimens for the degradation period range between 1802 and $2075 \mathrm{MPa}$, and those of miniature specimens between 2024 and $2446 \mathrm{MPa}$.

For the same degradation study, Fig. 8(a) and (b) illustrate $\sigma_{\mathrm{ys}}$ as a function of degradation time for both miniature and medium-sized specimens of PLLA and PDLLA respectively. Across the degradation period, $\sigma_{\mathrm{ys}}$ for medium-sized specimens were between 33.0 and 49.1 $\mathrm{MPa}$, and those of miniature specimens were between 33.2 and 47.9 $\mathrm{MPa}$. Both specimen sizes for PLLA exhibit a similar trend that consists of a $\sigma_{\mathrm{ys}}$ plateau region followed by a step-change decrease in $\sigma_{\mathrm{ys}}$ of approximately $30 \%$ after an accelerated degradation of 8 days and 13 days for medium and miniature beams respectively. Medium-sized PDLLA beams recorded a decrease in $\sigma_{\mathrm{ys}}$ of $30 \%$ by 5 days for degradation and remained relatively constant for up to 13 days. PDLLA miniature beams recorded comparable $\sigma_{\text {ys }}$ ranging between 40.3 and 47.7 MPa throughout the degradation period.

\section{Discussion}

When comparing the average $E$ of miniature beams with that of medium-sized beams measured using different equipment in both

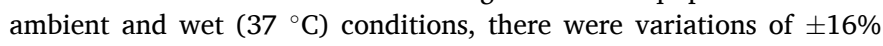
across all materials. Concentrating on AFTF measurements, miniature beams recorded a change in average $E$ between $2 \%$ and $14 \%$ when
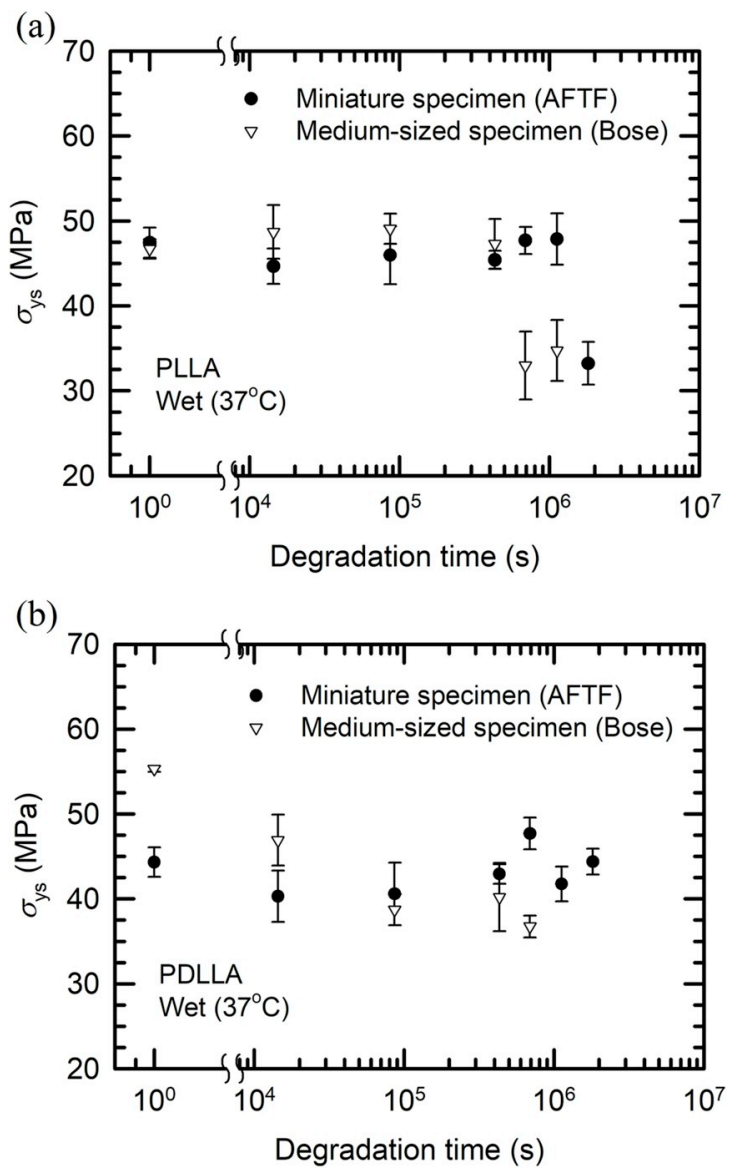

Fig. 8. Flexural strength of medium-sized and miniature specimens for (a) PLLA and (b) PDLLA measured at $37 \mathrm{C}$ in water, determined with two different equipment, after an accelerated degradation period of up to 21 days in PBS at 50 C. Results at $1 \mathrm{~s}$ are control samples that have not experienced hydrolytic degradation. 
compared to medium-sized counterparts for all materials at ambient conditions. Although the discrepancy is small, a plausible explanation is centred around the errors arising from the specimens' dimensional accuracy. Similar observations were made in Petisco-Ferroro et al.'s work on compression of polymeric micropillars [13]. Medium-sized beams are moulded directly to shape, and exhibit shrinkage effects during the cooling step of compression moulding. This is most noticeable as a curvature on the top and bottom faces, as shown in the cross-section in Fig. 9. The consequence of this is manifested in the assumption of a rectangular cross-section in the calculation of the second moment of area, consequently affecting the determination of mechanical properties. Although post-moulding machining could have been attempted for both specimen sizes to achieve more precise geometries, this would have encountered clamping challenges due to the reduced size, and likely required annealing steps to relieve residual stresses. For these reasons, the direct moulding technique was employed as a compromise manufacturing process that reliably produces homogenous and isotropic samples within a reasonable time frame [8]. Similar shrinkage effects were not observable for miniature beams, probably because these were obtained by cutting from larger moulded sheets, and hence the curvature due to shrinkage was less pronounced. Measuring the dimensions of miniature specimens was more challenging owing to their small size, and a greater relative measurement error was present.

Beyond the linear elastic region, the stress in medium-sized beams tested with AFTF reduced after $\sim 3 \%$ strain, as illustrated in Fig. 5(a) and (b), whereas miniature specimens displayed a continuously increasing stress. The change in specimen geometry from medium to miniature size led to $\sigma_{\mathrm{ys}}$ of PLLA and PDLLA to deviate by $12 \%$ and $14 \%$ respectively, as reported in Table 2 . This apparent increase in $\sigma_{\mathrm{ys}}$ for miniature beams can be attributed to the error in the cross-section arising from the shrinkage effects in the medium-sized beams. This leads to an overestimation of the second moment of area (due to the flat micrometer contacts), and hence an underestimation of the yield stress. It is worth noting that in the PS shown in Fig. 5(c) there is acceptable agreement between the miniature and medium-sized specimens for the reduced strain range, partly due to the reduced strain range and partly to the less pronounced shrinkage.

When polylactides are tested in water at $37 \mathrm{C}$, the mechanical properties can be influenced by two additional factors: the speed of the uptake of water, and the influence of water on craze formation. In a previous study, water uptake measurements carried out by monitoring mass change indicated that medium-sized PDLLA specimens reached $\sim 85 \%$ of the saturation level at $37 \mathrm{C}$ within 24 hours [14], and saturated to $\sim 1 \%$ water by mass. Based on scaling laws and assuming 1-dimensional diffusion, the same level of saturation is estimated to occur in the miniature specimens after $\sim 1$ hour of water immersion. This timescale is far closer to the timescale of the flexural experiment with the miniature beams, and therefore is consistent with the reduction in stiffness observed for wet testing (without degradation) in the PDLLA

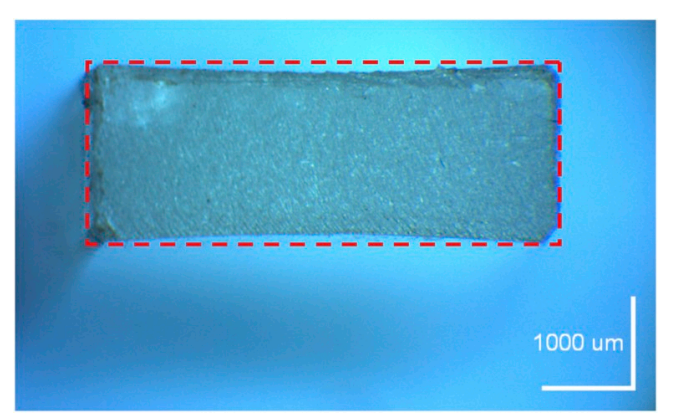

Fig. 9. Cross-section at the centre region of a medium-sized PLLA specimen demonstrating the shrinkage effect after compression moulding. The expected rectangular shape of the cross section is artificially highlighted with a dashed line. beams. Crazing was observed to have taken place in tested PDLLA medium-sized beams. It is reasonable to postulate that the presence of water acts either as a mild plasticizer or simply to reduce the energy required to form new surface as required by craze formation [15]. Hence the water is likely to have lowered the craze initiation stress more quickly for miniature specimens than for the larger specimens, contributing to a reduction in $\sigma_{\mathrm{ys}}$. The same effects were not observed in the PLLA beams, possibly due to the initial crystallinity.

When considering accelerated degradation in PBS at $50 \mathrm{C}$, the moduli measurements obtained with different equipment and specimen size for each material were broadly in agreement for the first 5 days, within experimental error. The step change increment in $E$ of semicrystalline PLLA for both medium-sized and miniature specimens after 5 days and 13 days respectively is likely to be related to the dramatic onset of crystallisation, which occurs at different time points in the bars of different dimensions. Additionally, the medium-sized beams recorded an earlier decrease in $\sigma_{\mathrm{ys}}$ relative to the miniature beams. During the accelerated degradation, the degradation products accumulate within the medium-sized beams and promote an autocatalytic reaction that further degrades the polymer. The formation of crystals requires mobility in the polymer chains, which is a consequence of the release of constraints arising from degradation. The degradation of polylactides is acid-catalysed, and hence unusually faster in the thicker beams where the acid by-products of degradation take longer to diffuse out into the buffer solution [14]. The reduction in PLLA specimen size delayed the significant decrease in $\sigma_{\mathrm{ys}}$ by $\sim 13$ days whilst reduction in PDLLA retained similar $\sigma_{\mathrm{ys}}$ throughout the degradation period of 21 days. This suggests that where diffusion is sufficiently fast the PBS solution can neutralise the degradation products, and hence suppress the autocatalytic process [14]. The PDLLA exhibited no such rise in $E$, which decreased to a plateau and remained approximately constant until the samples were too brittle for flexural testing. The plateau region could be attributed to the presence of $\alpha$-crystals that form as degradation progresses, as observed in X-ray diffraction measurements of degraded medium-sized PDLLA beams [14].

Overall, the AFTF has demonstrated the ability to characterise mechanical properties of miniature specimens in both ambient conditions and immersed in a fluid at elevated temperatures. It was shown that the AFTF is capable of characterising mechanical behaviour consistently with conventional test equipment. The AFTF can measure properties in 3-point bending on specimens weighing less than $10 \mathrm{mg}$ and is capable of testing at a range of specimen dimensions that facilitates investigation of material phenomena themselves affected by size. One limitation of the AFTF is the practical challenge of accurately producing and measuring dimensions of miniature beam specimens prior to testing owing to their reduced size. When working with polymers, specimen production and measurement is always a challenge, and in this work, it has affected the medium-sized beams in different ways to the miniature beams.

\section{Conclusions}

An adaptable flexural testing fixture (AFTF) has been designed for testing small polymeric specimens using a linear actuator to provide accurate controllable vertical displacement of the loading nose, small load cells to measure the response, and specimen supports suitable for both miniature and medium-sized beam geometries. The construction of the AFTF and calibration procedures to assess actuator displacement, load measurement and fixture compliance have been described. The AFTF has been shown capable of characterising the flexural properties of polymeric materials in both ambient and controlled temperature liquid conditions using miniature specimens weighing less than $10 \mathrm{mg}$, but is also capable of testing medium-sized specimens weighing less than $1 \mathrm{~g}$.

Validation of the AFTF was performed by measuring the flexural properties of a range of crystalline and amorphous polymeric materials and comparing the measurements against properties obtained using 
conventional test equipment. Benchmark flexural measurements were performed with medium-sized specimens of dimensions $\begin{array}{lll}60 & 5 & 2\end{array}$ $\mathrm{mm}^{3}$, while miniature specimens of dimensions $7 \quad 2 \quad 0.5 \mathrm{~mm}^{3}$ were employed on the AFTF. Corrections for shear effects and bending rotations at the support rollers, as well as for rig compliance, were applied to the data.

A comparison of flexural properties of materials in ambient and in water at $37 \mathrm{C}$ obtained with the AFTF and with two conventional test machines showed generally good agreement. Small discrepancies were obtained when comparing data between medium-sized and miniature specimens, and were attributed to imprecise specimen geometries due to shrinkage effects. In water, properties of semi-crystalline PLLA were relatively independent of specimen size, possibly due to the initial crystallinity, whereas properties of amorphous PDLLA exhibited a decrease $E$ and $\sigma_{\mathrm{ys}}$ when the specimen size was reduced. This was attributed to the faster uptake of water in the miniature specimens, and to the effect of water on the surface energy, and hence on craze initiation.

The fixture was also used to monitor properties of polylactides subjected to accelerated hydrolytic degradation in PBS solution at $50 \mathrm{C}$ for various time intervals. For each grade of polylactide, the general trends of $E$ and $\sigma_{\text {ys }}$ from miniature specimens as a function of degradation time were similar to those observed on larger specimens, but degradation phenomena influencing the mechanical properties occurred at different times. Here, the specimen dimensions were found to affect the accumulation of acidic degradation by-products and influence the autocatalytic degradation process differently. The miniature specimens' reduced thickness allowed diffusion to neutralise the acid by-products sufficiently fast to suppress autocatalysis, whereas the properties of medium-sized specimens manifested the effects of autocatalytic degradation.

The evidence shown suggests that testing miniature specimens with AFTF can provide useful information about the constitutive response of polymers at the milligram scale with the versatility of ambient and wet testing, but that care must be taken both with specimen preparation and with specific phenomena that are affected by specimen size.

\section{Data availability}

Data presented in this study is available upon request.

\section{Declaration of competing interest}

The authors declare that they have no known competing financial interests or personal relationships that could have appeared to influence the work reported in this paper.

\section{CRediT authorship contribution statement}

Gabriel Y.H. Choong: Conceptualization, Methodology, Formal analysis, Software, Data curation, Writing - original draft, Writing - review \& editing, Visualization. Alessia Canciani: Investigation, Validation, Software, Data curation. Davide S.A. De Focatiis: Conceptualization, Methodology, Software, Resources, Supervision, Writing - review \& editing, Funding acquisition.

\section{Acknowledgements}

The authors would like to acknowledge Ms. Anne-Lise Brisse for help with selected flexural measurements, and Mr. Paolo Parolin for images of specimen cross-sections. This work was supported by the Engineering and Physical Sciences Research Council, United Kingdom, grants EP/ J017272/1 and EP/N019784/1.

\section{References}

[1] En ISO 178:2019, Plastics - Determination of Flexural Properties, British Standards Institution, 2019.

[2] C. Doerffel, G. Jüttner, R. Dietze, Micro test specimens for compound engineering with minimum material needs, Mater. Sci. Forum 825-826 (2015) 928-935.

[3] D. Drummer, A. Seefried, S. Meister, Characterization of material stiffness on injection moulded microspecimens using different test methods, Adv. Mater. Sci. Eng. 2014 (2014) 1-8.

[4] G. Lewis, Key issues involved with the use of miniature specimens in the characterization of the mechanical behavior of polymeric biomaterials-a review, J. Biomed. Mater. Res. 63 (2002) 455-466.

[5] J.L. Kuhn, S.A. Goldstein, R. Choi, M. London, L. Feldkamp, L.S. Matthews, Comparison of the trabecular and cortical tissue moduli from human iliac crests, J. Orthop. Res. 7 (1989) 876-884.

[6] R.P.S. Chaplin, A.J.C. Lee, R.M. Hooper, M. Clarke, The mechanical properties of recovered PMMA bone cement: a preliminary study, J. Mater. Sci. Mater. Med. 17 (2006) 1433-1448.

[7] C.I. Albert, J. Jameson, G. Harris, Design and validation of bending test method for characterization of miniature pediatric cortical bone specimens, Proc. Inst. Mech. Eng. H 227 (2013) 105-113.

[8] D.S.A. De Focatiis, Tooling for near net-shape compression moulding of polymer specimens, Polym. Test. 31 (2012) 550-556.

[9] D.S.A. De Focatiis, C.P. Buckley, Determination of craze initiation stress in very small polymer specimens, Polym. Test. 27 (2008) 136-145.

[10] F. Mujika, On the effect of shear and local deformation in three-point bending tests, Polym. Test. 26 (2007) 869-877.

[11] I.W. Gilmour, A. Trainor, R.N. Haward, Elastic moduli of glassy polymers at low strains, J. Appl. Polym. Sci. 23 (1979) 3129-3138.

[12] Moldflow Material Testing Report Natureworks Pla, Moldflow Plastics Labs, Australia, 2007. Retrieved on: 1/4/2019, https://www.natureworksllc.com/ /me dia/Technical_Resources/Properties_Documents/PropertiesDocument_7000DMol dFlowReport_pdf.pdf.

[13] S. Petisco-Ferrero, R. Cardinaels, L.C.A. van Breemen, Miniaturized characterization of polymers: from synthesis to rheological and mechanical properties in $30 \mathrm{mg}$, Polymer 185 (2019) 121918.

[14] M. Ward, Mechanical and Structural Performance of Melt-Processable Bioresorbable Engineering Nanocomposites, PhD Thesis, University of Nottingham, Nottingham, United Kingdom, 2018.

[15] D.S.A. De Focatiis, C.P. Buckley, L.R. Hutchings, Roles of chain length, chain architecture, and time in the initiation of visible crazes in polystyrene, Macromolecules 41 (2008) 4484-4491. 